\title{
Hacia una definición del concepto grupo de interés
}

\section{Diego Solís Delgadillo*}

Perfiles Latinoamericanos, 25(50)

2017 | pp. 83-101

DOI: $10.18504 / \mathrm{pl} 2550-005-2017$

\section{Resumen}

El concepto grupo de interés se utiliza para referirse a actores muy diversos, lo que evita la comparación de resultados entre investigaciones. Así pues, se enfrenta un problema de acumulación que proviene de complicaciones en la definición de conceptos básicos. En este trabajo se comparan los usos del concepto grupo de interés analizando las ventajas, limitaciones e implicaciones empíricas de cada caso. Al final se propone identificar los elementos centrales de dicho concepto, lo que ayuda a solucionar el problema de acumulación.

\begin{abstract}
The concept interest group is used to make reference to a wide set of actors, which inhibits the comparison of results among studies. Therefore, these studies face a problem of accumulation which derives from definitional problems of its core concepts. This text compares the different uses of the concept interest group, it analyses the advantages, limitations and empirical implications of each use of the term. Finally, it intends to identify the core elements of the concept interest group that could help to confront the problem of accumulation.
\end{abstract}

Palabras clave: conceptos, ciencia política, grupos de interés, participantes de presión, poder político de facto, stakeholders.

Keywords: Concepts, political science, interest groups, pressure participant, de facto political power, stakeholders.

* Candidato a Doctor de Investigación en Ciencias Sociales con mención en Ciencia Política por la Facultad Latinoamericana de Ciencias Sociales, Sede México (Flacso México). Maestro en Asuntos Políticos y Políticas Públicas por El Colegio de San Luis A. C. | diego.solis@flacso.edu.mx 


\section{Introducción ${ }^{1}$}

$\mathrm{D}$

urante los primeros años de la ciencia política, el estudio de los grupos de interés ocupó un lugar central en esa disciplina. Trabajos clásicos como los de Truman (1951), Key (1964 [1956]) y Dahl (1961) se enmarcaron en la corriente pluralista, cuyo centro de atención eran los grupos de interés y cómo estos actuaban en el sistema de presión. Sin embargo, esta línea de análisis fue desplazada por investigaciones de temas como los sistemas electorales, los tipos de gobierno y los regímenes políticos. De ese modo, mientras la ciencia política ha hecho importantes avances en numerosos tópicos, el estudio de los grupos de interés parece modesto.

En este sentido, Baumgartner \& Leech (1998) identifican obstáculos como la orientación parroquial de muchas investigaciones, la falta de información sobre las actividades en que estas se involucran y el problema con la definición de sus conceptos básicos. Así, la falta de una comprensión compartida de lo que es un grupo de interés dificulta la acumulación del conocimiento. Es decir, que conceptos fundamentales de este campo de estudio, como grupo de interés e influencia, contienen confusiones que tienen consecuencias sobre la información recolectada y los hallazgos empíricos. Ello ha provocado que cada autor haga referencia a objetos de estudio distintos, lo que evita la comparación de resultados y la acumulación de conocimiento (Baumgartner \& Leech, 1998).

Este trabajo ofrece una definición mínima de grupo de interés. Para ello se revisa la literatura identificando los elementos comunes y buscando diferenciar dicho concepto de sus vecinos semánticos. El artículo se ha estructurado de la siguiente forma: en el primer apartado se expone por qué los conceptos importan en la investigación social; en el segundo se detallan algunas consecuencias empíricas de distintas conceptualizaciones; en el tercero se revisa la literatura evaluando las ventajas y limitaciones de los diferentes usos del concepto tema de este análisis, y se identifican los elementos conceptuales comunes presentando que distingue al término grupos de interés en relación con otros cercanos. Finalmente, se presentan las conclusiones.

\section{Los conceptos como contenedores de datos}

Como apunta Schedler (2010), los conceptos en ciencias sociales son con frecuencia complejos y disputados. Por eso la investigación científica no puede

1 El autor agradece las sugerencias de los dictaminadores, así como los comentarios de Andrea Pozas Loyo, que contribuyeron sustancialmente en la elaboración de este trabajo. 
dar por sentado que los demás miembros de la comunidad los comprenden de la misma manera. Por lo tanto, es necesario explicitar los compromisos conceptuales de los que se parten, es decir, que se requiere de transparencia para determinar con exactitud a qué fenómenos se hace referencia.

La claridad conceptual es un punto importante pues permite o inhibe la comparación de resultados. De tal forma que si un concepto se define con incongruencia se puede obstaculizar la acumulación de conocimiento, ya que, en lugar de estudiar un mismo fenómeno, se puede estar investigando a varios con el mismo nombre (Schedler, 2010). Un ejemplo es el concepto Estado fallido, que puede ser comprendido desde el colapso del gobierno hasta la debilidad del Estado para hacer cumplir la ley. Estas definiciones inciden sobre lo que se puede clasificar como Estado fallido y dependiendo de la conceptualización de este se puede llegar a conclusiones distintas sobre su origen y consecuencias. En suma, el concepto puede estar refiriéndose a fenómenos distintos.

De lo anterior se desprende una regla fundamental: la conceptualización consiste en que una palabra se debe referir a un solo significado (Sartori, 2012). La confusión surge cuando varias palabras se usan para un solo concepto (sinonimia) o una palabra conduce a múltiples significados (homonimia). En el estudio de los grupos de interés aparecen ambos problemas: existen significados distintos de lo que es un grupo de interés y también una amplia gama de términos para designar al mismo fenómeno, esto es, hay ambigüedad (Sartori, 2012; Schedler, 2010).

La multiplicidad de usos del término grupo de interés va desde autores que reservan dicho concepto para organizaciones voluntarias que presentan demandas frente al Estado, pasando por definiciones que incluyen a organizaciones privadas como empresas o think tanks, hasta quienes consideran a los gobiernos locales como grupos de interés. Tal diversidad también se refleja en los neologismos que se utilizan para referirse a los grupos de interés, entre ellos grupos de presión, intereses creados, intereses organizados, intereses específicos o asociaciones de interés.

\section{Respuestas en la literatura al problema conceptual de grupo de interés}

Las dificultades de la multiplicidad de significados del concepto grupo de interés han sido identificadas previamente por autores como Jordan et al. (2004) y Beyers et al. (2008). Los primeros abogan por una restricción de ese concepto a "asociaciones voluntarias". La solución en este caso radica en excluir las organizaciones que no enfrentan dilemas de acción colectiva. En consecuencia, 
la solución sería aumentar la intención y limitar la extensión del concepto. Por su parte, Beyers et al. (2008) coinciden en reservar dicho concepto a grupos conformados por membresías voluntarias y proponen la noción organizaciones de interés para los demás actores que buscan incidir sobre el sistema político, pero sosteniendo que unos y otros pueden ser comparables en su función de cabildeo.

Ahora bien, resulta importante señalar que la restricción del concepto grupo de interés a asociaciones voluntarias es muy costosa para el área de estudio ya que acota el número de observaciones y reduce la capacidad de generalización de los resultados para todo el sistema de presión. Esto último es en especial importante dado que una de las motivaciones centrales del estudio del sistema de presión es conocer cómo actores externos a las instituciones democráticas inciden sobre las decisiones colectivas. Al excluir a actores como empresas, universidades o think tanks, se pierde gran parte de las dinámicas del sistema de presión e incluso se llega a resultados sesgados. Más aún cuando se observa que los hallazgos empíricos han mostrado que los grupos empresariales son los que más inciden en la toma de decisiones (Dür et al., 2013).

El principal argumento para una definición restringida es que esta permite contar con grupos que son muy similares y por eso más fácilmente comparables en sus indicadores. Sin embargo, estudios recientes han utilizado definiciones funcionales de grupo de interés y han sido capaces de comparar el éxito de distintos tipos de grupos; por ejemplo, Dür et al. (2014) encuentran que las asociaciones voluntarias han sido más exitosas en el cabildeo en la Unión Europea que los grupos empresariales, mientras que Grossman \& Pyle (2013) demuestran que entre mayor es el número de grupos de interés - independientemente de si son asociaciones voluntarias o no- que presionan sobre una comisión legislativa con respecto a un proyecto de ley, habrá más posibilidades de su aprobación.

En este texto se sostiene que para que un grupo de interés sea considerado como tal es necesario que ejerza presión sobre las decisiones públicas, lo que contrasta con Jordan et al. (2004) y Beyers et al. (2008), para quienes la asociación voluntaria es la condición necesaria de ese concepto. Por otra parte, si bien la presión política es una condición esta no es suficiente para clasificar a un actor como grupo de interés, para ello tres elementos adicionales lo distinguen de sus vecinos semánticos: 1) que los grupos cuenten con una estructura estable en el tiempo, 2) que las presiones ejercidas se den por medios no violentos, y 3) que no busquen responsabilidades gubernamentales. La introducción de estos elementos permite distinguir los grupos de interés de conceptos como movimiento social, partido político o grupos terroristas o antisistema. 


\section{Consecuencias empíricas del concepto grupo de interés}

La conceptualización que sirve de base a una investigación tiene consecuencias empíricas pues, como sostiene Sartori (2012), los conceptos son contenedores de datos, de manera que diferentes conceptualizaciones conducen a capturar distinta información. A continuación se ejemplifican varios problemas generados por una conceptualización muy limitada que incrementa los atributos que debe tener una organización para ser considerada grupo de interés y, por otro lado, una definición laxa, que permite que una amplia gama de casos puedan ser considerados como grupo de interés.

En su estudio del acceso de los grupos de interés a distintas arenas, Binderkratz et al. (2014) parten de definir a los grupos de interés como "organizaciones con membresías que trabajan para obtener influencia política”. Con una explicación así se excluye a buena cantidad de actores que son parte del sistema de presión y que no cuentan con membresías voluntarias, como corporaciones, agencias de cabildeo, universidades, hospitales y think tanks.

El objetivo de dichos autores es determinar en qué medida el tipo de grupo incide sobre el acceso a determinadas arenas políticas (parlamento, burocracia, medios de comunicación), y llegan a la conclusión de que los resultados son mixtos. No obstante, no es posible conocer si estos últimos son producto de la selección de casos, específicamente de la omisión de organizaciones que no enfrentan dilemas de acción colectiva, pero que acceden a dichas instancias.

Para muestra de todo lo que excluye una definición basada en membresías voluntarias basta con revisar los hallazgos de Schlozman (2010) sobre la conformación del sistema de presión en Estados Unidos. Dicho trabajo clasifica las 27000 organizaciones presentes entre 1981 y 2006 en el Directorio de Washington, un registro de todas las organizaciones involucradas en actividades de lobby. Schlozman encontró que solo una pequeña parte de esas organizaciones son grupos con membresías y que la mayoría de los actores activos en Washington son corporaciones. De tal forma que si el interés de la investigación es saber cómo grupos específicos afectan la representación política, asumir una definición restrictiva provoca que se pierda gran parte de la información acerca de lo que pasa en el sistema de presión.

Por el contrario, una definición laxa, como las utilizadas en estudios de economía política - por ejemplo, Grossman \& Helpman (2001), para quienes un grupo de interés es cualquiera que comparta características y preocupaciones en un conjunto de temas-, lleva a un referente empírico diferente del de Scholzman (2010), pues no solo incluye a los participantes en el sistema de presión sino que asume como grupo de interés a segmentos del electorado como los obreros, hispanos, mujeres, etc. En consecuencia, incluye a grupos que pueden 
no estar movilizados en el sistema de presión, en gran parte porque el interés de investigación se encuentra en el ámbito electoral.

Por tanto, asumir distintas conceptualizaciones puede conducir a diferentes resultados acerca de qué tan influyente es el grupo de interés. Más aún, si el propósito del investigador es conocer en qué medida aquel afecta la toma de decisiones difícilmente podrá comparar los resultados empíricos de una definición restringida con otra para la que el término es sinónimo de segmento social. Dada esta diversidad de conceptualizaciones es pertinente llevar a cabo una revisión que identifique los elementos esenciales del concepto grupo de interés.

\section{¿Cómo arribar a una conceptualización de grupo de interés?}

Con la finalidad de arribar a un concepto de grupo de interés en esta sección se procede a una revisión de la literatura, o lo que Sartori llama "reconstrucción" semántica. Con este ejercicio se espera identificar los elementos esenciales del concepto que sean suficientes para diferenciarlo de otros términos, procurando que no excluya fenómenos en general vistos como su extensión (Gerring, 2012). En otras palabras, en esta parte se explora cuál es el centro semántico del concepto, y para ello se identificarán los elementos compartidos por todas o la mayoría de las definiciones de grupo de interés (Schedler, 2010).

Asimismo, se analizan las ventajas y limitaciones de cada uno de los conceptos ofrecidos por la literatura, así como sus implicaciones empíricas. Finalmente se ubica al concepto dentro de su campo semántico para establecer sus fronteras respecto de nociones vecinas, ya que para conocer una especificidad es necesario averiguar qué comparte un concepto con otros y qué lo separa de ellos (Schedler, 2010). En este análisis se distingue tres formas en que ha sido entendido un grupo de interés: 1) como asociación que comparte actitudes, 2) como organización voluntaria, y 3) como lobby. A continuación se revisa cada caso.

\section{Grupo de interés como asociaciones con actitudes compartidas}

Entre las conceptualizaciones centradas en el rasgo de actitudes compartidas se halla la de Truman (1951: p. 37), autor que define a grupo de interés como "cualquier grupo, que con base en una o más actitudes compartidas, realiza ciertos reclamos sobre otros grupos en la sociedad para el establecimiento, mantenimiento o acrecentamiento de formas de comportamiento que están implícitos en las actitudes compartidas". 
Hay dos aspectos en tal definición: a) que la asociación mantenga actitudes compartidas, y b) que realice reclamos sobre otros grupos. Así, esta explicación permite la inclusión tanto de grupos organizados como de los latentes. Esto desde el supuesto de que cuando las decisiones públicas afectan los intereses de los grupos latentes estos se movilizan en la arena pública. En específico, Truman incluye de este modo a distintos grupos en general excluidos del estudio de la toma de decisiones, por ejemplo, las asociaciones de campesinos o profesionales.

No obstante, la definición de Truman presenta un importante problema: sus fronteras son borrosas respecto a otros términos como el de movimiento social, en el que se pueden identificar tanto actitudes compartidas como reclamos sobre el gobierno. Desde el enfoque de Truman esto calificaría como grupo de interés, sin embargo, se puede argumentar que estas movilizaciones no lo son porque carecen de una estructura que les dé estabilidad en el tiempo; en otras palabras, un grupo de interés se caracteriza por cierto grado de institucionalización.

En este sentido, mientras el movimiento social se singulariza por su espontaneidad, el grupo de interés lo hace por una estructura que le da estabilidad e identidad en el tiempo. Por ejemplo, no es lo mismo la lucha por los derechos civiles en Estados Unidos que la National Association for the Advancement of Colored People (NAACP); la primera consistió en movilizaciones espontáneas en favor de los derechos de los afroamericanos, la segunda es una organización formalmente establecida que brega por los derechos de dicha minoría. Los movimientos sociales pueden dar pie a la formación de grupos de interés, pero no son sinónimos. En particular porque el objetivo de los estudios de los grupos de interés es explicar la incidencia de agrupaciones estructuradas en la representación política; en tanto que los estudios de los movimientos sociales se concentran en la participación política espontánea y en el involucramiento directo de los ciudadanos en el espacio público.

Un segundo problema en la definición de Truman es que ella sostiene que las demandas pueden ser hechas sobre otros grupos y no exclusivamente sobre el Estado. Por tanto, se puede asumir que todos los reclamos entre grupos particulares podrían constituir un grupo de interés. $\mathrm{Al}$ respecto, es pertinente acotar el término a los grupos que mantienen relaciones con el Estado, debido a que el interés de la ciencia política se orienta a la incidencia de estos actores sobre las decisiones que son vinculantes para todos los miembros de la sociedad.

La necesidad de centrarse en los grupos que mantienen relaciones con el Estado ha sido reconocida por autores que utilizan el concepto de Truman como, por ejemplo, Hrebenar \& Morgan (2009), quienes añaden que una condición del grupo de interés es que esté involucrado en el sistema político y busque 
afectar la política pública. Una definición que incluye elementos funcionales que se abordarán más adelante. En suma, el concepto de Truman es impreciso porque sus fronteras son borrosas, debido a que sus características son insuficientes para identificar sus referentes empíricos y, en consecuencia, carece de poder discriminatorio y se traslapa con otros conceptos.

Ahora bien, la vaguedad de los conceptos de grupo de interés no es exclusiva de la corriente pluralista. Tal hecho es también común en los estudios de economía política, en especial en aquellos sobre comportamiento electoral inclinados a atribuir actitudes a segmentos o estratos de la sociedad. Esto lo muestra el trabajo de Grossman \& Helpman (2001: p. 105), autores para los que un grupo de interés "especial" se refiere a "cualquier grupo de ciudadanos que comparte características identificables y preocupaciones similares en un conjunto de temas". La idea que subyace en esta definición es que estos grupos enarbolan intereses distintos a los del votante mediano.

Partiendo de lo anterior, Grossman \& Helpman (2001) consideran como grupos de interés a segmentos sociales como los miembros de una misma profesión o de una industria, o de una minoría racial o los adherentes a un mismo credo. Al dotar teóricamente a estos grupos de actitudes e intereses compartidos, dichos autores los introducen en el teorema del votante mediano. De tal manera que esta conceptualización sirve para modelar a estos grupos y tratar de ponderar cómo ellos pueden alejar a los candidatos o tomadores de decisiones de las preferencias del votante mediano. Esta conceptualización se encuentra también en otros estudios sobre comportamiento electoral como los de Morton (1991) y Gilens \& Page (2014); ambos tienen como base modelos en los que los individuos pueden ser clasificados en grupos mutuamente excluyentes y en los que los miembros de cada grupo comparten preferencias idénticas sobre política pública.

Esta definición de grupo de interés es aún más vaga, dado que el único elemento que sirve de fundamento a dichos autores sería que comparte características o preocupaciones sobre determinado tema. Siguiendo esta definición, todo grupo social podría clasificarse como de interés, aun cuando no se involucre en actividades políticas, es decir, que incluso los grupos latentes teorizados por Truman podrían ser grupos de interés. Un problema en esta definición es que los elementos considerados son insuficientes para diferenciar el grupo de interés de actores no movilizados en el sistema de presión. Más aún, cuando no se considera que los grupos realicen demandas o que estos se encuentren organizados. En realidad estos autores se refieren a segmentos del electorado y a cómo las decisiones políticas responden más a uno que a otro. Asumirlos como grupos de interés induce a un problema de ambigüedad ya que multiplica los significados del concepto. 
Un segundo conjunto de definiciones retoma la idea de las actitudes compartidas, pero añade nuevos elementos que hacen más complejo el concepto. En este caso se ha puesto atención en el carácter voluntario, de tal forma que la característica fundamental de los grupos de interés es que cuentan con membresías voluntarias organizadas formalmente en torno a un tema específico (Jordan et al., 2004). En esta conceptualización se identifican dos subtipos: los que reservan el término a las asociaciones con membresías individuales, y los que además incluyen a las asociaciones de organizaciones.

En el primer subtipo se encuentra la propuesta de LaPalombara (1964: p. 18), quien considera que los grupos de interés son "cualquier agregación de individuos interactuantes que manifiestan deseos consistentes con respecto a la colocación autoritativa de valores". O la de Walker (1991), para quien un grupo de interés se identifica con las asociaciones abiertas a membresías y preocupadas por aspectos específicos de la política pública.

Para ambos autores existen tres elementos fundamentales en el concepto de grupos de interés: 1) se trata de asociaciones de membresías individuales y voluntarias, 2) se encuentran organizados alrededor de objetivos o valores, y 3) estas asociaciones presentan sus intereses en el espacio público. Estas definiciones se han utilizado sobre todo para el estudio de asociaciones civiles preocupadas por temas específicos de la agenda pública, e incluirían organizaciones de la sociedad civil como la National Rifle Association (NFA) o Greenpeace. Se trata sobre todo de organizaciones formalmente constituidas y fincadas en un conjunto de individuos que superan dilemas de acción colectiva y presentan sus demandas ante el Estado.

Sin embargo, Salisbury (1984) identificó que, además de los grupos con membresías individuales, hay otros a los que llamó "grupos de instituciones" de los que son ejemplo las cámaras empresariales, y los grupos de universidades o gobiernos locales. Todos comparten las características de aglutinarse en torno a un tema de preocupación para sus miembros y por su intención de presentar demandas ante el Estado. No obstante, a diferencia de las membresías individuales son sus dirigentes — dueños de las empresas, rectores, gobernadores—, los que deciden unirse a estos grupos y no todos los individuos que conforman la organización. Más aún, estas organizaciones en lo individual no cuentan con miembros, en el sentido de afiliaciones voluntarias, sino que se trata de trabajadores, estudiantes o burócratas, cuya motivación para unirse a dichas organizaciones no gira alrededor de una agenda política.

En resumen, estas definiciones introducen el elemento de una estructura basada en membresías voluntarias, ponen en el centro de la organización las 
preocupaciones políticas y el hecho de que estos intereses sean presentados en el espacio público. Sin duda, las organizaciones que a estos autores les interesa estudiar cumplen con esas características; tanto las membresías individuales como las de grupos de organizaciones.

Sin embargo, aun así el concepto se puede traslapar con otros. Por ejemplo, organizaciones terroristas como Al Qaeda o el Estado Islámico son organizaciones con fines políticos en las que sus miembros ingresan voluntariamente y se les encuentra en el espacio público mediante actos violentos que buscan incidir en la toma de decisiones. Con los elementos de las definiciones de arriba se podría considerar a ambos como grupos de interés, y lo mismo podría suceder con agrupaciones antisistema, como las guerrillas o las separatistas, que recurren a la violencia con fines políticos. En consecuencia, los elementos de tales definiciones son insuficientes para distinguir entre grupos de interés y dichas organizaciones. No obstante un rasgo que podría contribuir a delimitar un grupo de interés respecto de grupos ilegales o antisistémicos son los medios no violentos.

Por otro lado, esta conceptualización también puede confundirse con la definición de partido político, ya que todas las características se cumplen: son organizaciones con membresías voluntarias en forma de afiliados, con intereses compartidos como ideologías o programas partidistas, y expresan sus demandas dentro del sistema político. Sin embargo, hay algo que distingue a los partidos políticos y que no se encuentra en los grupos de interés, estos no pretenden asumir responsabilidades gubernamentales. Esto último genera una discusión sobre la idea de Salisbury (1984), autor que asume como grupo de interés a las asociaciones de gobiernos locales y a funcionarios electos.

\section{Grupos de interés como lobby}

Un tercer bloque de estudios se ha centrado en las funciones de los grupos. En este caso, la característica central que distingue a los grupos de interés es que se vean involucrados en actividades de cabildeo.

Una de las primeras definiciones de este tipo es la de Key (1964 [1956]: 18), quien entiende a los grupos de interés como "asociaciones que tienen una función representativa que comunica los deseos de sus miembros a las autoridades". Por su parte, Knoke (1986: 2) parte de la noción de asociación, a la que define como "un grupo formalmente organizado, cuyos miembros, ya sean personas u organizaciones, no son financieramente recompensadas", y señala que estas son grupos de interés cuando "intentan influir en las decisiones gubernamentales". 
A primera vista tal definición se podría confundir con la de partido político, por lo que Key añade que, a diferencia de aquel, los grupos de interés son asociaciones que promueven sus agendas sin hacerse responsables del manejo del gobierno. Al respecto, cabe destacar que los primeros estudios de este tipo distinguen claramente entre los actores estatales y los grupos de interés. Sin embargo, los estudios de política pública, preocupados por la influencia de la burocracia y los gobiernos locales, fueron estirando el concepto para incluir a dichos actores como grupos de interés. Por ejemplo, el trabajo de Lindblom (1991 [1980]) incluye a los funcionarios de gobierno y a los departamentos o agencias gubernamentales, bajo la lógica de que si están involucrados en actividades de lobby, también se trata de grupos de interés. Esta confusión se origina en que equipara el término stakeholder, ampliamente utilizado en política pública, como sinónimo de grupo de interés. De ahí que consideren a todos los actores con intereses en juego en la toma de decisiones como grupos de interés.

El trabajo de Salisbury (1984) sigue la misma lógica; su argumento es que tras revisar las actividades de cabildeo en Estados Unidos se puede observar que los actores más exitosos en actividades de lobby no son grupos de membresías voluntarias sino "instituciones". Con ese punto de partida elabora una clasificación que divide entre grupos de interés con miembros y sin miembros. Esto le permite introducir en el análisis a actores excluidos en corporaciones, universidades, hospitales y gobiernos locales. De aquí se desprende que estudios como el de Heinz et al. (1993), Gray \& Lowery (1996) y Schlozman (2010) tomen como referentes empíricos las bases de datos sobre cabilderos registrados; el razonamiento es que si están registrados así, entonces son organizaciones que se pueden considerar como grupos de interés.

Con frecuencia el objetivo de estos estudios ha sido demostrar el sesgo de representación en el sistema de presión. En otras palabras, evidenciar que son los grupos empresariales, y especialmente las grandes empresas, las que ejercen mayor influencia en la toma de decisiones.

Esta clasificación ha recibido críticas, por ejemplo, Jordan et al. (2004) rechazan la idea de "grupos sin miembros", ya que consideran que las membresías voluntarias son un aspecto esencial del concepto grupo. Sostienen que si bien tanto un grupo de interés como una corporación están constituidos por individuos, el tipo de relaciones de estos con sus organizaciones son profundamente distintas. Consideran así que un elemento crucial para calificar a una organización como "grupo" es si este enfrenta dilemas de acción colectiva.

Entre los grupos considerados dentro de la definición funcional se presenta un particular problema con los gobiernos locales, esto debido a que uno de los elementos que distingue a los grupos de interés es que no buscan asumir 
responsabilidades de gobierno. Para Salisbury (1984) y Lindblom (1991 [1980]), aquellos pueden ser considerados como grupos de interés cuando en realidad son dos tipos diferentes de actores que pertenecen al proceso de toma de decisiones. Ahora bien, si las actividades de cabildeo son un rasgo definitorio se puede llegar a excesos como el considerar que los cuerpos diplomáticos son grupos de interés. Por eso el carácter no gubernamental que introduce Key (1964 [1956]) es una característica del grupo de interés que permite diferenciarlo de otros términos.

A pesar de las críticas a la idea de "grupos sin miembros" de Salisbury, esta clasificación ha sido ampliamente utilizada. Ello quizá pueda entenderse porque en los estudios sobre lobby se encuentra que las grandes empresas como Google, General Electric o Exxon Mobil son las que invierten más recursos en el sistema de presión. Por lo tanto, puede considerarse que excluirlas de las investigaciones dejaría un hueco en las organizaciones que inciden en la toma de decisiones.

\section{Propuestas restrictivas del concepto grupo de interés}

Dos trabajos han tratado de resolver la confusión conceptual de grupo de interés. El primero es de Jordan et al. (2004), quienes critican las definiciones funcionales y proponen reservar ese término para asociaciones con membresías, es decir, para las que enfrentan dilemas de acción colectiva. Por eso proponen clasificar a todas las organizaciones que presionan al gobierno como "participantes de presión", las que a su vez subdividen en "grupos de interés-presión" y "participantes de política”. La diferencia entre estos radica en que los primeros enfrentan dilemas de acción colectiva, y los segundos son asociaciones sin miembros como corporaciones u universidades.

Caben dos observaciones a la propuesta de Jordan et al. (2004). En primer lugar, las subdivisiones están fundadas en diferencias organizacionales, pero la idea de "participantes de presión" implícitamente reconoce que parte de la definición es funcional, ya que lo que caracteriza a los "participantes de presión" son sus intentos de incidir en la toma de decisiones; en segundo lugar, no queda claro qué se gana creando conceptos distintos para los "participantes de presión”. En términos sustantivos lo que estos autores realizan es una clasificación entre grupos con membresías voluntarias y organizaciones cuyos objetivos rebasan la arena política.

Un segundo intento por clarificar el concepto grupo de interés es el de Beyers et al. (2008), autores que proponen un concepto con tres elementos: 1) organización, que implica la agregación de preocupaciones de individuos u organizaciones; 2 ) intereses políticos, que se refiere a los intentos de estas organizaciones 
para influir en los resultados políticos, y 3) informalidad, esto es, que estas asociaciones no buscan ocupar cargos públicos o competir en elecciones, sino que persiguen sus objetivos por medio de interacciones informales con políticos o burócratas.

Respecto de organizaciones como corporaciones, gobiernos locales o universidades, que estrictamente no agregan preferencias, Beyers et al. (2008) las refieren como "organizaciones de interés". En otras palabras, reconocen que, si bien cuentan con intereses públicos y no buscan responsabilidades institucionales de gobierno, ${ }^{2}$ carecen del central elemento de la agregación de preferencias, aunque al mismo tiempo señalan que son "equivalentes" a los grupos de interés vistos desde su función de influenciar al gobierno. En consecuencia, a pesar de diferenciar entre grupo de interés y organizaciones de interés, aceptan la idea de Salisbury de grupos sin miembros.

En la propuesta de Beyers et al. (2008) se observan elementos organizacionales y funcionales, es decir, tanto el requisito de organización como la función de abogacía de los grupos. Asimismo, rescata la idea de Key (1964 [1956]) respecto a que estos grupos no buscan responsabilidades gubernamentales. Esta definición dota de mayores elementos para distinguir en relación con otro tipo de actores en el sistema político como los partidos políticos, las organizaciones de interés y los movimientos sociales, pero al mismo tiempo no incluye los canales por los que se lleva a cabo la abogacía. Así pues, si se acepta la propuesta de Beyers et al. (2008) descartando que las demandas sean transmitidas por medios no violentos, entonces diversos grupos ilegales como los extremistas podrían incluirse como grupos de interés. En eso consiste la importancia de incluir este elemento.

\section{Elementos compartidos y vecinos conceptuales}

La revisión de la literatura nos permite identificar los elementos compartidos por todas las definiciones expuestas hasta aquí. A pesar de que cada una enfatiza en diferentes aspectos, como los intereses compartidos, las membresías voluntarias o las funciones de representación de intereses de los grupos, existen elementos comunes entre las definiciones. En el cuadro 1 se comparan los elementos presentes en diez definiciones de grupo de interés, incluyendo las de Jordan $e t$ al. (2004) y Beyers et al. (2008).

2 Esta característica no estaría presente en el caso de los gobiernos locales, pero aun así los autores incluyen en ella el lobby intragubernamental. 
Cuadro 1. Elementos compartidos por las definiciones de grupo de interés

\begin{tabular}{|c|c|c|c|c|}
\hline \multirow[b]{2}{*}{ Autor } & \multicolumn{4}{|c|}{ Elementos del concepto } \\
\hline & $\begin{array}{l}\text { Intereses/valores } \\
\text { compartidos }\end{array}$ & $\begin{array}{c}\text { Membresías } \\
\text { voluntarias }\end{array}$ & $\begin{array}{l}\text { Representación } \\
\text { de intereses }\end{array}$ & $\begin{array}{c}\text { Carácter no } \\
\text { gubernamental }\end{array}$ \\
\hline Truman (1951) & $x$ & & $x$ & \\
\hline $\begin{array}{l}\text { Hrebenar \& Morgan } \\
\text { (2009) }\end{array}$ & $x$ & & $x$ & \\
\hline $\begin{array}{l}\text { Grossman \& } \\
\text { Helpman (2001) }\end{array}$ & $\mathrm{x}$ & & & \\
\hline LaPalombara (1964) & $x$ & $x$ & $\mathrm{x}$ & \\
\hline Walker (1991) & $x$ & $x$ & & \\
\hline Key (1956) & & & $x$ & $\mathrm{x}$ \\
\hline Knoke (1986) & & $\mathrm{x}$ & $x$ & \\
\hline Salisbury (1984) & & & $\mathrm{x}$ & \\
\hline Jordan et al. (2004) & & $\mathrm{x}$ & $x$ & \\
\hline Beyers et al. (2008) & & $x$ & $x$ & $x$ \\
\hline
\end{tabular}

Fuente: Elaboración propia.

Del cuadro 1 se concluye que el elemento más frecuente es la representación de intereses. Todas las definiciones, excepto las de Grossman \& Helpman (2001) y Walker (1991), incluyen los intentos de estos grupos por incidir en las decisiones públicas, por medio de reclamos (Truman, 1951), buscando afectar la política pública (Hrebenar \& Morgan (2009), manifestando deseos sobre la colocación autoritativa de valores (LaPalombara, 1964), como organización que comunica los deseos de sus miembros a las autoridades (Key, 1956) o como organizaciones que intentan incidir en las decisiones gubernamentales (Salisbury, 1984; Knoke, 1991). El común denominador son las funciones que desempeña el grupo de interés, por ello se puede decir que tal es el centro semántico del concepto.

Por lo tanto, la revisión apunta a que un elemento esencial en el concepto grupo de interés es la representación de intereses frente al Estado. En principio esto apoyaría el uso de una definición funcional del concepto. No obstante, como se ha mostrado, este aspecto no es suficiente porque no permite distinguir de otros grupos que intentan incidir sobre la toma de decisiones. Para poder delimitar la idea se requiere conocer su campo semántico, lo que implica ubicar otros conceptos vecinos a grupo de interés y delimitar las fronteras de este respecto de aquellos. Esto significa identificar otros conceptos que se refieran a actores que busquen incidir en las decisiones estatales, entre estos, movimiento social, grupos antisistema, grupos terroristas, partidos políticos, lobby, poderes fácticos y stakeholders.

En primer lugar, los movimientos sociales comparten con los grupos de interés su propósito de afectar las decisiones públicas. No obstante, son fenómenos distintos en su organización, pues mientras el primero es espontáneo y carece de estabilidad en el tiempo, el segundo cuenta con una estructura institucional 
que le otorga este último rasgo. En consecuencia, algo que distingue a uno de otro es la institucionalización de la organización.

En segundo lugar, una serie de grupos intentan afectar las decisiones políticas mediante la violencia, como el caso de las agrupaciones antisistema o terroristas. Aquí la distinción fundamental son los medios con los cuales se presiona al Estado: el grupo de interés no presenta sus demandas con violencia. En tercero, los partidos políticos también buscan afectar las decisiones gubernamentales; al respecto es importante retomar la propuesta de Key (1956), quien sostiene que el grupo de interés no aspira a adquirir responsabilidades gubernamentales, no intentan conquistar cargos públicos.

De este ejercicio de contraste surgen tres elementos más que permiten caracterizar al grupo de interés: son organizaciones con estructuras institucionalizadas, actúan por medios no violentos y no buscan responsabilidades gubernamentales. Partiendo del centro semántico y de dichos elementos, podemos definir al grupo de interés como una organización formalmente estructurada que busca incidir a través de medios no violentos sobre la toma decisiones públicas sin asumir responsabilidades gubernamentales.

Esta definición permite incluir tanto a grupos de miembros voluntarios como a los de instituciones. Es una definición útil sobre todo para estudiar la representación política, esto es, para investigar en qué medida diversos actores inciden o no sobre la toma de decisiones. Asumir una definición restringida de grupo de interés, como proponen Jordan et al. (2004), tiene como consecuencia perder gran parte de la información de lo que sucede en el sistema de presión. Más aún, con esta definición se pueden realizar clasificaciones que permitirían comparar diferencias entre estrategias, recursos o efectividad de distintos tipos de grupos de interés, pero sin excluir a determinados grupos del campo de estudio.

Finalmente, otros conceptos son utilizados con frecuencia como sinónimos de grupo de interés, entre estos, lobby, poderes fácticos y stakeholders. Es decir, el concepto de grupo de interés se ha confundido tanto con actividades, como con recursos y otro tipo de actores del sistema político. En primer lugar se suele utilizar de modo indistinto lobby y grupo de interés, lo que es un error porque el primero se refiere a una actividad con la que determinados actores intentan persuadir a los tomadores de decisiones sobre un tema determinado. ${ }^{3} \mathrm{Si}$ bien los grupos de interés se pueden ver involucrados en actividades de lobby, la actividad y la organización no son lo mismo; además, estos no son los únicos actores en el sistema de presión inmersos en actividades de cabildeo, como lo demuestra el lobby intragubernamental.

3 Por ejemplo el concepto de lobby de Milbrath (1963) que lo define como "la estimulación y transmisión de una comunicación $[\ldots]$ dirigida a un decisor gubernamental con la esperanza de influenciar su decisión”. 
Una segunda confusión es denominar al grupo de interés como poder fáctico. Aquí se confunden características de la organización con la misma organización, ya que mientras poderes fácticos remite a las fuentes informales de poder, ${ }^{4}$ grupo de interés designa a una organización con características específicas. Acemoglu \& Robinson (2006) abordan el tema del poder político de facto e identifican tres de sus fuentes: recursos, capacidad de acción colectiva y violencia. Por ende, el poder político de facto puede ser ejercido por muy diversos actores: corporaciones, líderes sociales, guerrillas, terroristas o grupos de interés. De tal manera que grupos de interés y poderes fácticos no son sinónimos.

Finalmente, los términos stakeholder y grupo de interés suelen ser utilizados como sinónimos. El primero fue utilizado en su origen en el lenguaje corporativo y después adoptado en los estudios de política pública. Es un concepto que se refiere a "personas, grupos u organizaciones que deben ser tomadas en cuenta por los líderes, gerentes o personal de primera línea" (Bryson, 2004) o como "todas las partes que son afectadas o que afectan la estrategia de una organización" (Nutt \& Backoff, 1992). En este sentido, el concepto no se limita al espacio público, es decir, puede ser utilizado tanto al interior de organizaciones privadas como en los asuntos públicos; en contraste, el término grupo de interés se limita a considerar a actores activos en el espacio público. Al mismo tiempo, la palabra stakeholder no especifica las funciones de estos actores, de manera que indica que son afectados por las decisiones (en este caso públicas), pero no señala como algo inherente al concepto que presenten sus demandas frente al Estado, en otras palabras, no estipula que los stakeholder desempeñen una función representativa. ${ }^{5}$

\section{Conclusiones}

La diversidad de definiciones de grupo de interés puede dificultar la comparación de resultados de investigación debido a que conduce a diferentes referentes empíricos. Estos varían desde conceptualizaciones centradas en los intereses compartidos, pasando por las concentradas en las membresías voluntarias, hasta las que se enfocan en las funciones de los grupos.

Estudios previos como los de Jordan et al. (2004) y Beyers et al. (2008) han propuesto definiciones restringidas que limitan el uso del concepto a grupos que resuelven dilemas de acción colectiva y cuentan con membresías voluntarias, ya que de

4 Esto es, que el poder que ejercen no proviene de un puesto de dentro de las instituciones públicas.

5 Dentro de esta literatura aparece un problema conceptual similar al aquí analizado: Eden \& Ackerman

(1998) consideran que solo los actores con poder para afectar el futuro de la organización son stakeholders, mientras que Nutt \& Backoff (1992) y Bryson (2004) abogan por una definición que incluye a los actores "sin poder". 
acuerdo con estos autores una definición así permite una mejor comparación de resultados. No obstante, asumir una definición de este tipo resulta costoso debido a que excluye a gran parte de los actores presentes en el sistema de presión.

En contraste, partiendo de la revisión de la literatura, este trabajo ha identificado los elementos comunes entre las diferentes explicaciones sobre lo que son los grupos de interés, para ello ha analizado sus conceptualizaciones en distintas tradiciones buscando su centro semántico, esto es, los elementos básicos del concepto. Tras analizar diversas tradiciones conceptuales al respecto, se encuentra que el elemento en común es que el grupo de interés represente demandas en el espacio público, es decir, que el aspecto funcional es central en el concepto, el cual se encuentra incluso en definiciones restringidas como las de Jordan et al. (2004) y Beyers et al. (2008).

Ahora bien, tras una análisis de los conceptos vecinos se establecieron algunas fronteras que permiten diferenciar respecto de otros como movimiento social, grupos antisistema o terroristas y partidos políticos, fenómenos que pudieran ser considerados como grupos de interés según algunas de las definiciones aquí presentadas. $\mathrm{Al}$ respecto vale reiterar que el grupo de interés tiene como rasgos la organización, los canales no violentos y el carácter no gubernamental. Surge así su definición: una organización formalmente estructurada que busca incidir a través de medios no violentos en la toma de decisiones públicas sin asumir responsabilidades gubernamentales.

Esta delimitación de las fronteras con conceptos vecinos permite la comparación entre grupos partiendo de sus funciones, es decir, que a pesar de sus diferencias organizacionales pueden ser comparadas respecto a su efectividad en la representación de intereses; esta explicación permite preguntarse ¿qué tipos de grupos de interés son más eficaces en las actividades de cabildeo? o ¿qué tipo de estrategias son más exitosas en el sistema de presión? Esta clase de preguntas no pueden ser contestadas desde una definición restringida que excluya a grupos empresariales o instituciones que no enfrentan dilemas de acción colectiva. En suma, una definición como la de este trabajo es más propicia para estudiar la representación política, ello debido a que no excluye participantes del sistema de presión y, más importante aún, incluye el centro semántico que atraviesa a la mayoría de las conceptualizaciones de grupo de interés.

\section{Referencias}

Acemoglu, D. \& Robinson, J. A. (2006). Paths of Economic and Political Development. En Weingast, B. \& Wittman, D. (Eds.). The Oxford Handbook of Political Economy (pp. 673692). Nueva York: Oxford University Press. 
Baumgartner, F. R. \& Leech B. (1998). Basic Interests: The Importance of Groups in Politics and in Political Science. Nueva Jersey: Princeton University Press.

Beyers, J., Eising, R. \& Maloney, W. (2008). Researching Interest Group Politics in Europe and Elsewhere: Much We Study, Little We Know? West European Politics, 31(6), 1103-1128.

Binderkrantz, A. S., Christiansen, P. M. \& Pedersen, H. H. (2014). Governance: An International Journal of Policy, Administration and Institutions, 28(1), 95-112.

Bryson, J. M. (2004). What to do When Stakeholders Matter. Public Management Review, 6(1), 21-53.

Dahl, R. (1961). Who Governs: Democracy and Power in an American City. New Haven: Yale University Press.

Dür, A. \& Mateo, G. (2013). Gaining Access or Going Public? Interest Groups Strategies in Five European Countries. European Journal of Political Research, 52(5), 660-686.

Dür, A. \& Mateo, G. (2014). Public Opinion and Interest Group Influence: How Citizen Groups Derailed the Anti-Counterfeiting Trade Agreement. Journal of European Public Policy, 21(8), 1199-1217.

Eden, C. \& Ackerman, F. (1998). Making Strategy: The Journey of Strategic Management. Londres: Sage.

Gerring, J. (2012). Social Science Methodology: A Unified Framework. Nueva York: Cambridge University Press.

Gilens, M. \& Page, B. I. (2014). Testing Theories of American Politics: Elites, Interest Groups, and Average Citizens. Perspectives on Politics, 12(3), 564-581.

Gray V. \& Lowery, D. (1996). The Population Ecology of Interest Representation: Lobbying Communities in the American States. Michigan, Ann Arbor: Michigan University Press.

Grossman, G. \& Helpman, E. (2001). Special Interest Politics, Cambridge: The mIT Press.

Grossman, M. \& Pyle, K. (2013). Lobbying and Congressional Bill Advancement, Interest Groups and Advocacy, 2(1), 91-111.

Heinz, J. P., Laumann, E. O., Nelson, R. L. \& Salisbury, R. H. (1993). The Hollow Core: Private Interests in National Policymaking. Cambridge: Harvard University Press. 
Hrebenar R. J. \& Morgan, B. B. (2009). Lobbying in America: A Reference Handbook, Santa Bárbara: ABC Clio.

Jordan, G., Halpin D. \& Maloney, W. (2004). Defining Interest: Disambiguation and the Need for New Distinctions? British Journal of Politics and International Relations, 6(2), 1-8.

Key, V. O. (1964 [1956]). Politics, Parties and Pressure Groups, Nueva York: Thomas Y. Crowell.

Knoke, David, 1986, Associations and Interest Groups. Annual Review of Sociology, 12, 1-21.

LaPalombara, J. (1964). Interest Groups in Italian Politics. Nueva Jersey: Princeton University Press.

Lindblom, C. E. (1991 [1980]). El proceso de elaboración de politicas públicas. México: Porrúa.

Milbrath, L. W. (1963). The Washington Lobbyist. Chicago: Rand McNally.

Morton, R. (1991). Groups in Rational Turnout Models. American Journal of Political Science, 35(3), 758-776.

Nutt, P. \& Backoff, R. (1992). Strategic Management of Public and Third Sector Organizations: A Handbook for Leaders. San Francisco: Jossey Bass.

Salisbury, R. H. (1984). Interest Representation: The Dominance of Institutions. The American Political Science Review, 78(1), 64-76.

Sartori, G. (2012). Cómo hacer ciencia politica. México: Taurus.

Schedler, A. (2010). Concept Formation in Political Science. Documento de Trabajo, núm. 219, División de Estudios Políticos. México: Centro de Investigación y Docencia Económicas.

Schlozman, K. L. (2010). Who Sings in the Heavenly Chorus? The Shape of the Organized Interest System. En Maisel, S. L. \& Berry, J. (Eds.). The Oxford Handbook of American Political Parties and Interest Groups. Nueva York: Oxford University Press.

Truman, D. (1951). The Governmental Process: Political Interests and Public Opinion. Nueva York: Alfred A. Knopf.

Walker, J. L. (1991). Mobilizing Interest Groups in America. Michigan, Ann Arbor: University of Michigan Press.

Recibido el 12 de junio de 2015. Aceptado el 10 de enero de 2017. 